\title{
In-situ friction monitoring of self-mated CVD diamond coatings using acoustic emission
}

\author{
C. S. Abreu ${ }^{1, a}$, F. J. Oliveira ${ }^{2, b}$, J. R. Gomes ${ }^{3, c}$, M. Belmonte ${ }^{4, d}$, A.J.S. \\ Fernandes ${ }^{5, \mathrm{e}}$, R. F. Silva ${ }^{2, f}$ \\ ${ }^{1}$ Physics Dept., Porto Superior Engineering Institute, ISEP, 4200-072 Porto, Portugal \\ ${ }^{2}$ Ceramics and Glass Eng. Dept., CICECO, Univ. of Aveiro, 3810-193 Aveiro, Portugal \\ ${ }^{3}$ Mechanical Eng. Dept., CIICS, University of Minho, 4800-058 Guimarães, Portugal \\ ${ }^{4}$ Instituto de Cerámica y Vídrio (CSIC), Cantoblanco, 28049 Madrid, Spain \\ ${ }^{5}$ Physics Department, Univ. of Aveiro, 3810-193 Aveiro, Portugal \\ a csa@isep.ipp.pt, ${ }^{b}$ filipe@cv.ua.pt, ${ }^{c}$ jgomes@dem.uminho.pt, ${ }^{d}$ mbelmonte@icv.csic.es, \\ e toze2@fis.ua.pt, ${ }^{\top}$ rsilva@cv.ua.pt
}

Keywords: CVD diamond; Silicon nitride; Friction behaviour; Acoustic emission

\begin{abstract}
In-situ measurements of acoustic emission (AE) in self-mated tribological pairs of CVD diamond coated silicon nitride $\left(\mathrm{Si}_{3} \mathrm{~N}_{4}\right)$ were made with the purpose of investigating the relationship between AE signal and friction events. A good correlation is found between the energy dissipation/emission processes, therefore enabling the possibility of monitoring the different friction regimes occurring during the sliding contact of microcrystalline diamond (MCD) coatings. Deposition of MCD on flat and ball-shaped $\mathrm{Si}_{3} \mathrm{~N}_{4}$ samples was accomplished using microwave plasma assisted chemical vapour deposition (MPCVD) with $\mathrm{H}_{2} / \mathrm{CH}_{4}$ gas mixtures. The friction behaviour of self-mated MCD coatings was assessed using a reciprocating ball-on-flat geometry. The tests were run in ambient atmosphere without lubrication, the frequency $(1 \mathrm{~Hz})$ and stroke $(6 \mathrm{~mm})$ were kept constant while the applied normal load varied in the range $10-80 \mathrm{~N}$. The microstructure, surface topography and roughness of the MCD coatings were characterised by SEM and AFM techniques. The diamond quality was assessed from micro-Raman spectroscopy. The friction evolution was characterised by a short running-in period where the main feature is a sharp peak reaching values as high as approximately 0.6 followed by a steady-state regime with very low values in the range $0.03-0.04$.
\end{abstract}

\section{Introduction}

Acoustic emission (AE) is a phenomenon originating from a rapid relaxation of strain energy produced by deformation, part of which radiates as a short time stress wave that can be detected by an appropriate transducer, coupled to the source surface [1].

Research has been undertaken into the use of AE technology in monitoring the material response to tribological strain, principally on how significant the emission from sliding pairs is and how it relates to parameters governing the behaviour of tribosystems [2]. A relationship was found between the acoustic phenomenon and the dissipation of mechanical energy that can be used to classify different friction contacts [3].

Homologous contacts of chemical vapour deposition (CVD) diamond coated films are characterised by a wide range of friction coefficient values, depending on the sliding conditions. Even in ambient atmosphere, which gives very low equilibrium friction values, a variation of more than one order of magnitude could be found between the steady-state value and the starting friction coefficient. This wide range makes difficult analysing the friction behaviour in terms of prevailing friction events or mechanisms, due to physical limitations in the measuring range of load cells. Thus, in-situ friction monitoring by AE could allow an easier detection of the distinct friction regimes or sliding events. 
The aim of this investigation was to study the use of AE for condition monitoring the sliding contact of self-mated microcrystalline diamond (MCD) coatings, submitted to high applied loads, in a dry sliding reciprocating arrangement.

\section{Experimental setup and test procedure}

Dense silicon nitride $\left(\mathrm{Si}_{3} \mathrm{~N}_{4}\right)$ substrates were prepared from powder mixtures of $\alpha$-Si $\mathrm{S}_{3} \mathrm{~N}_{4}(\mathrm{Starck}$ grade $\mathrm{M} 11$ ), $\mathrm{Y}_{2} \mathrm{O}_{3}$ (Starck grade $\mathrm{C}$ ) and $\mathrm{Al}_{2} \mathrm{O}_{3}$ (ALCOA CT-3000SG) with, respectively, the following proportions (wt.\%): 89.3/7.0/3.7. Following a procedure of homogenisation and sieving, the mixed powders were consolidated by uniaxial pressing (30 MPa), followed by isostatic pressing $(200 \mathrm{MPa})$. Pressureless sintering in a conventional graphite furnace $\left(1750^{\circ} \mathrm{C}, 2 \mathrm{~h}\right)$, was used to produce fully dense ( $>99 \%$ of the theoretical density) disc-shaped $\mathrm{Si}_{3} \mathrm{~N}_{4}$ substrates. All the sintered samples were ground in order to achieve the final dimensions of $10 \mathrm{~mm}$ of diameter and thickness of $3 \mathrm{~mm}$. Commercial balls (Kema Nord) of $\mathrm{Si}_{3} \mathrm{~N}_{4}$ with $5 \mathrm{~mm}$ diameter were also used as substrate material for the counter-bodies. In order to achieve adequate adhesion level and to enhance nucleation density, the flat specimens were submitted to surface pre-treatments with diamond paste polishing $(15 \mu \mathrm{m})$, followed by manual scratching with $0.5-1 \mu \mathrm{m}$ sized diamond powder on a silk cloth. A more thorough description of the processing of $\mathrm{Si}_{3} \mathrm{~N}_{4}$ substrates can be found elsewhere [4].

The deposition of MCD films was carried out in a microwave plasma chemical vapour deposition (MPCVD) system (ASTeX PDS 18, Seki Technotron Corp.). The deposition parameters used for all tested samples were as follows: microwave power $=3 \mathrm{~kW}$; chamber pressure $=1.2 \times 10^{4}$ $\mathrm{Pa} ; \mathrm{H}_{2} / \mathrm{CH}_{4}$ gas flow $=400 / 25 \mathrm{sccm}$; deposition time $=2.5 \mathrm{~h}$.

The tribological response of the MCD coated $\mathrm{Si}_{3} \mathrm{~N}_{4}$ films was assessed by a ball-on-flat tribometer (PLINT TE67/R). Self-mated sliding tests were conducted using a reciprocal dry sliding arrangement in ambient atmosphere $(\sim 50-60 \% \mathrm{RH})$. The diamond coated flat specimens were fixed to an oscillating table and made to contact with diamond coated balls (upper specimen), firmly attached to a sample holder arm. All the tests were performed with a constant stroke $(6 \mathrm{~mm})$ and frequency $(1 \mathrm{~Hz})$, while static applied normal loads in the range $10 \mathrm{~N}$ to $80 \mathrm{~N}$ were applied directly over the ball specimens. Considering a Hertzian elastic contact model, these loads produced initial pressures of 5-10 GPa [5].

The friction force was measured by means of a load cell, its signal amplified and acquired by a PC. Before each test, the load cell was calibrated by applying external dead-weights in the range of the measured loads. The sliding distance varied between $2 \times 10^{-2} \mathrm{~m}$ and $86 \mathrm{~m}$ in order to have a systematic account of the friction phenomenon, focused at different phases of the sliding interaction.

The AE signal was recorded during the sliding tests by a piezoelectric transducer (Brüel \& Kjaer) tightly fixed to the disc sample holder, in a position perpendicular to the sliding direction. An amplification/filtering (Brüel \& Kjaer) stage, with a frequency window of $50 \mathrm{KHz}-2 \mathrm{MHz}$, was used to convert the acoustic pressure intensity into a 0-10 V DC signal, at adequate acquisition rates by means of an A/D conversion board (Keithley PCMCIA 12 AIH). The diamond quality of the deposited films was studied by ex-situ micro-Raman spectroscopy using a $514.5 \mathrm{~nm} \mathrm{Ar}^{+}$incident laser beam, with a spot size of $\sim 1 \mu \mathrm{m}$ and few tens of $\mathrm{mW}$ power at the surface. Atomic force microscopy (AFM, Digital Instruments NanoScope IIIa) was used to study the surface topography and roughness of as-deposited and worn MCD coatings. Surface roughness values were assessed using inherent NanoScope III software, by acquiring three AFM fields with a scan size of 50x50 $\mu \mathrm{m}^{2}$. The surface microstructure, diamond grain size and film thickness were analysed by a Hitachi (model S-4100) scanning electron microscope (SEM).

\section{Results and discussion}

The most conventional morphology of CVD-grown diamond thin films corresponds to pyramidal micro-crystallites with well defined triangular (111) facets and a preferential $<100>$ crystal growth 
orientation [6]. The SEM photomicrograph in Fig. 1a, taken from a diamond coated $\mathrm{Si}_{3} \mathrm{~N}_{4}$ flat specimen, is a clear example of that morphology.

All the CVD diamond coated samples exhibited a fully adhered and continuous aspect. The average diamond grain size and film thickness of the MCD coatings, obtained from stereological and cross-sectional image analysis of higher magnifications SEM photomicrographs, showed values of $2.4 \pm 1.1 \mu \mathrm{m}$ and $\sim 12-14 \mu \mathrm{m}$, respectively.

A representative AFM micrograph of an as-deposited MCD flat sample is presented in Fig. 1b. As it can be seen, the films present a hilly topography combined with the presence of some deep valleys. These rugged surfaces were characterised by surface roughness parameters of $R_{a} \sim 170 \mathrm{~nm}$, $\mathrm{R}_{\mathrm{z}} \sim 1450 \mathrm{~nm}$ and maximum peak-to-valley height of $\sim 1480 \mathrm{~nm}$.
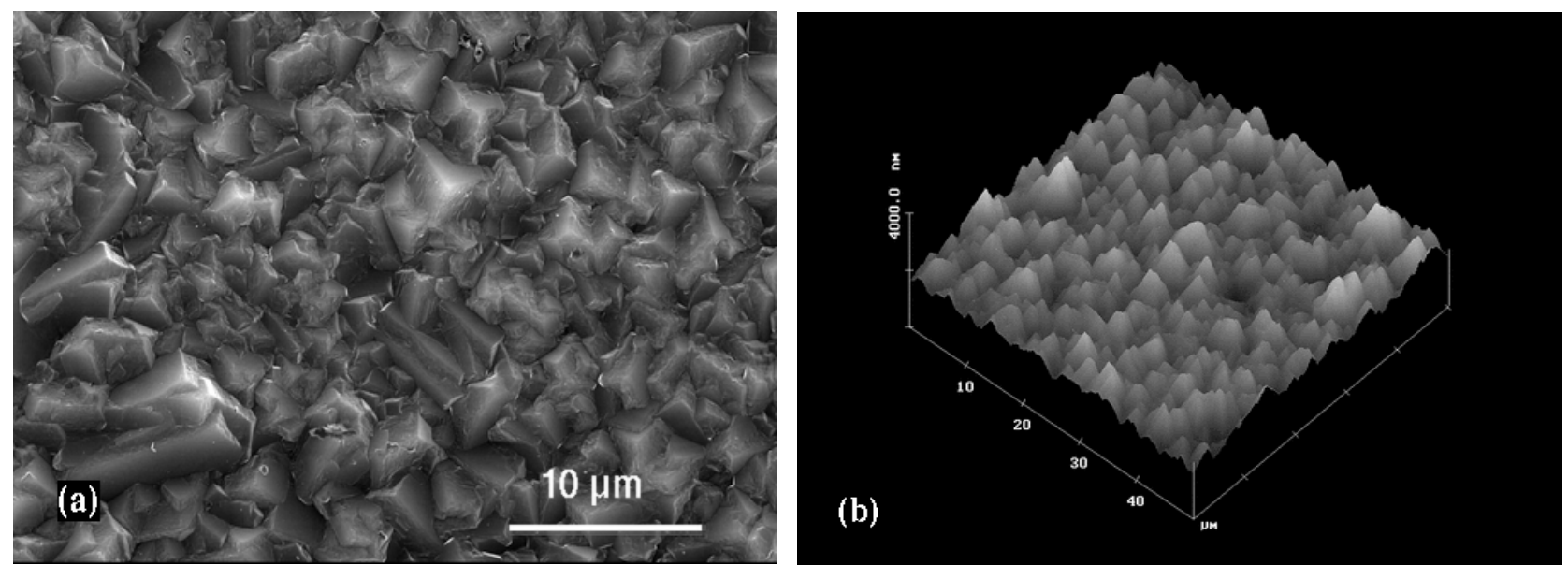

Fig. 1: As-deposited diamond coated $\mathrm{Si}_{3} \mathrm{~N}_{4}$ films: (a) SEM micrograph of representative $\mathrm{C}\{111\}-$ micro-pyramidal film on flat samples; (b) AFM image of typical flat specimens.

Fig. 2a shows a micro-Raman spectrum of the diamond films deposited onto flat specimens. The presence of a sharp peak centred around the line of natural diamond $\left(1332 \mathrm{~cm}^{-1}\right)$ and no evidence of broad graphitic bands ( $\mathrm{sp}^{2} \mathrm{C}-\mathrm{C}$ bonding), confirms the high quality and purity of the deposited films, while showing a stress-free state.

A typical friction coefficient evolution curve for self-mated MCD films is depicted in Fig. $2 b$. The plot reports to an applied load of $80 \mathrm{~N}$ and shows, as its main feature, a pronounced initial peak with a friction coefficient of $\sim 0.60$, followed by a gradual decay to very low friction coefficient values. These different behaviours for the friction coefficient are identified in the plot according to three distinct regimes (labelled I, II, III). Regime I results from the initial intense mechanical interaction between diamond asperities of contacting surfaces. Furthermore, it was observed that the magnitude of this sharp peak, for films with the same starting surface roughness, depends on the applied load, which corroborates a friction mechanism based on the interlocking of asperities. Such dependency is also found in the literature [7]. As sliding progressed the friction coefficient was significantly affected by the truncating and gradual blunting of diamond tip asperities. As such, regime II is related to a running-in effect associated to the accommodation of mating surfaces. The experimental results also showed that the length of this transition regime depended on the applied load. Afterwards, and for the remaining length of the sliding tests, the friction coefficient levels-off and enters into a steady-state phase (regime III), characterised by very low values $(\sim 0.03-0.04)$, this time as a result of the interaction between flattened diamond asperities and the minor contribution of adhesion for the overall friction mechanism in ambient atmosphere [8]. Regime III is also characterized by very low surface roughness values. AFM measurement yielded $\mathrm{R}_{\mathrm{a}} \sim 27 \mathrm{~nm}$ after tribological tests run under an applied load of $40 \mathrm{~N}$ for $86 \mathrm{~m}$. 

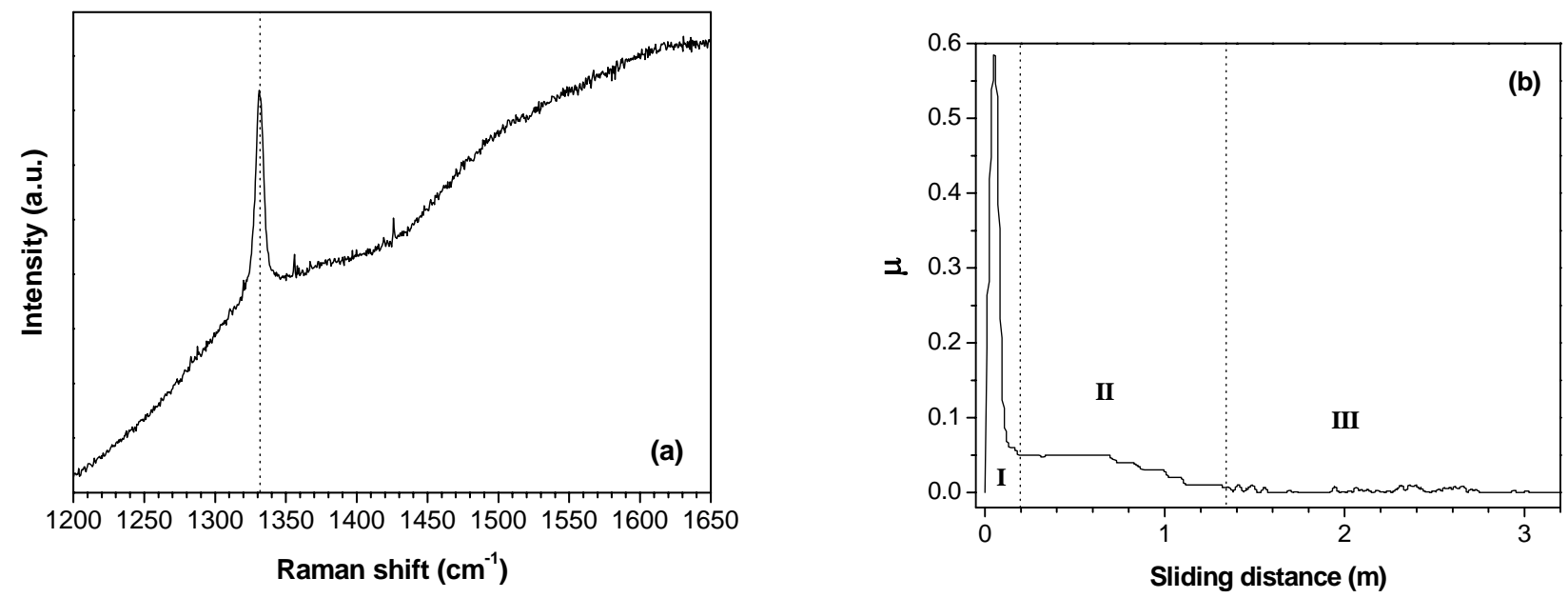

Fig. 2: Characterisation of MCD coated $\mathrm{Si}_{3} \mathrm{~N}_{4}$ films. (a) micro-Raman spectrum of as-deposited coatings, revealing a sharp peak centred at the diamond frequency line $\left(1332 \mathrm{~cm}^{-1}\right)$; (b) representative friction curve evolution showing the different friction regimes (applied load of $80 \mathrm{~N}$ ).

The relation between the sliding distance run immediately after completion of regime I, taken from the friction curves, and the duration of the AE signal burst corresponding to the friction peak is represented in the graph of Fig. 3a, as a function of the applied load. The AE signal burst was considered extinct when its amplitude had fallen below the background noise threshold level. As can be seen, a good correlation exists between the two curves stating that a process of energy dissipation driven by friction mechanisms of asperity interlocking/truncating is intimately related to a process of acoustic emission. This was accompanied by the production of an intense strident sound that lasted for a few initial reciprocating cycles $(<20)$. In addition, the plotted curves reveal an almost linear variation of the sliding distance run after ending of regime I, with increasing applied load, until $55 \mathrm{~N}$. Afterwards, and up to the critical load for film delamination $(>80 \mathrm{~N})$, both curves suffer little changes.

Similarly, the relation between the sliding distance run at the end of regime II and the duration of the corresponding AE signal burst is depicted in Fig. 3b, for different applied loads.
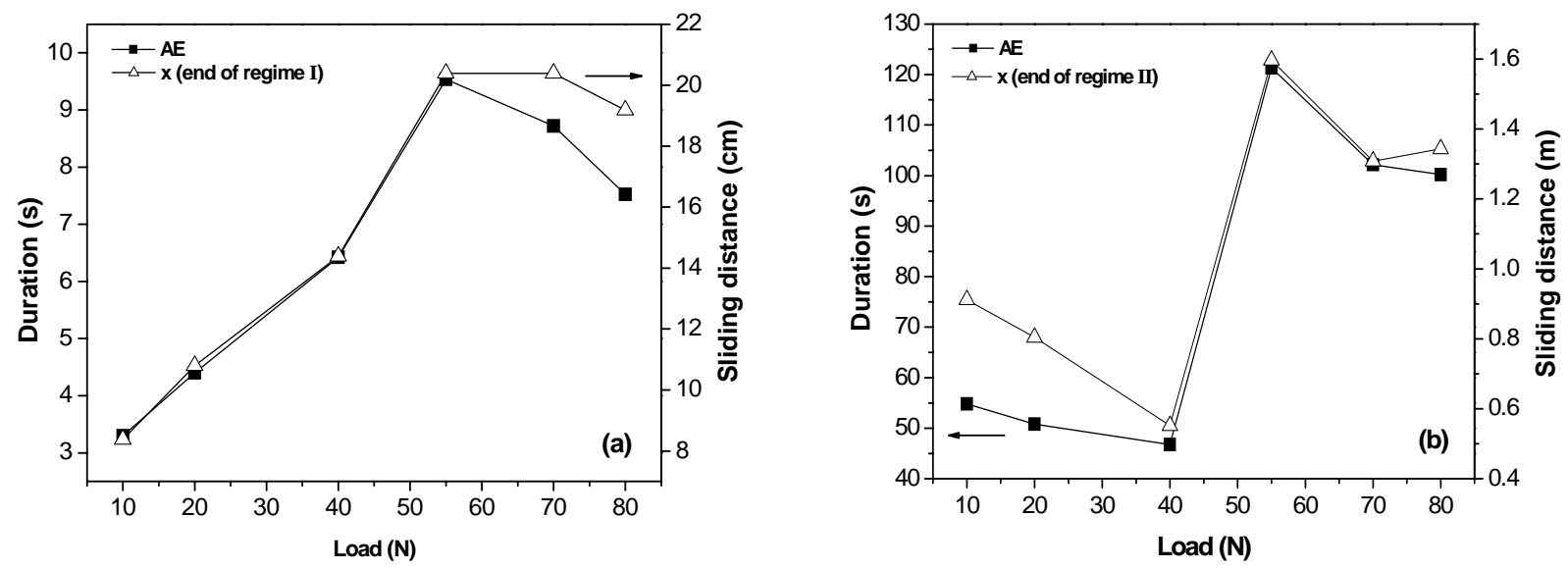

Fig. 3: Correlation between the sliding distance run at the end of (a) regime I and (b) regime II and the duration of AE signal burst, for various applied loads.

The correlation between the two curves is significant, which opens the possibility to clearly identify the distinct sliding regimes of MCD films by means of AE signal monitoring. Another feature 
shown in Fig 3b, is that steady-state sliding condition is achieved sooner for an intermediate applied load of $40 \mathrm{~N}$, i.e. the accommodation between opposing surfaces ends after a shorter sliding distance. A possible explanation for this could be related to a competing effect between the contact pressure level and the subsequent rate of surface smoothening by the blunting of asperities translated in a shorter or longer running-in regime - and some surface degradation by diamond pullouts observed for the higher loads, which delays further the accommodation of mating surfaces.

\section{Conclusions}

The friction behaviour of $\mathrm{MCD}$ coated $\mathrm{Si}_{3} \mathrm{~N}_{4}$ films revealed a characteristic running-in regime denoting an intense initial friction peak, followed by a short transition period, which in turns leads to very low equilibrium friction coefficients $(\sim 0.03-0.04)$.

A correlation exists between the AE signal and the frictional behaviour of sliding contacts. The friction peak at beginning and its evolution is closely followed by the AE signal.

A steady-state response is achieved sooner for an intermediate load $(40 \mathrm{~N})$. This relates to a competing effect between the rate of the blunting of asperities and surface degradation by diamond pull-outs. This confirms the possibility of monitoring the distinct friction regimes by AE techniques.

\section{Acknowledgements}

C.S. Abreu acknowledges PRODEP III funds supporting his PhD work. M. Belmonte acknowledges the financial support of the "Ramón y Cajal" program (MEC, Spain). The financial support of project POCTI/CTM/45423/2002 is gratefully acknowledged.

\section{References}

[1] J. Miettinen and V. Siekkinen: Wear Vol. 181-183 (1995), p. 897

[2] S. Lingard and K.K. Ng: Wear Vol. 130 (1989), p. 367

[3] T. Skåre and F. Krantz: Wear Vol. 255 (2003), p. 1471

[4] M. Belmonte, A.J.S. Fernandes, F.M. Costa, F.J. Oliveira and R.F. Silva: Diam. Relat. Mater. Vol. 12 (2003), p. 733

[5] P.D. Warren: J. Euro. Ceram. Soc. Vol. 15 (1995), p. 385

[6] K. Mallika and R. Komanduri: Thin Solid Films Vol. 396 (2001), p. 145

[7] K. Miyoshi: Mater. Sci. Eng. A Vol. 209 (1996), p. 38

[8] M.N. Gardos: Surf. Coat. Tech. Vol. 113 (1999), p. 183 